\title{
Propulsion System Dynamic Modeling of the NASA Supersonic Concept Vehicle for AeroPropulsoServoElasticity
}

\author{
George Kopasakis ${ }^{1}$, and Joseph W. Connolly ${ }^{2}$, Jonathan Seidel ${ }^{3}$ \\ NASA Glenn Research Center, Cleveland, Ohio 44135
}

\begin{abstract}
A summary of the propulsion system modeling under NASA's High Speed Project (HSP) AeroPropulsoServoElasticity (APSE) task is provided with a focus on the propulsion system for the lowboom supersonic configuration developed by Lockheed Martin and referred to as the $\mathrm{N}+2$ configuration. This summary includes details on the effort to date to develop computational models for the various propulsion system components. The objective of this paper is to summarize the model development effort in this task, while providing more detail in the modeling areas that have not been previously published. The purpose of the propulsion system modeling and the overall APSE effort is to develop an integrated dynamic vehicle model to conduct appropriate unsteady analysis of supersonic vehicle performance. This integrated APSE system model concept includes the propulsion system model, and the vehicle structural-aerodynamics model. The development to date of such a preliminary integrated model will also be summarized in this report.
\end{abstract}

\section{Introduction}

$\mathrm{T}$ HE slender configuration of low-boom aircraft, combined with rigid body effects and non-linear aerodynamics, often results in highly complex nonlinear aeroelastic/flight dynamics phenomena. Combined with the propulsion system, the AeroServoElastic ASE/APSE dynamic coupling phenomena and the resulting structural oscillations can affect the ride quality, and the vehicle flight dynamics and control. The APSE dynamic effects can simultaneously influence the airframe and propulsion system controls to produce undesirable effects on performance and flying characteristics. Thus, these APSE phenomena need to be thoroughly understood for supersonic flight to be safe, efficient and comfortable. Understanding these phenomena through the modeling capabilities that are being developed will provide the opportunity to use active controls to mitigate these undesirable effects in order to improve performance such as ride quality, vehicle stability, and flight efficiency. This new research will enhance the analysis and design capabilities for slender supersonic aircraft.

A top priority for the High Speed Project ASE task is to develop the tools required to perform accurate, high fidelity Aeroelastic (AE), ASE, and APSE analyses in support of the design of future low-boom supersonic civil aircraft. As a means of accomplishing that priority, the NASA HSP is working with Lockheed Martin to develop vehicle concepts and analyze the performance of these vehicles. Under the NASA N+2 (2 generations from present state) design, Lockheed Martin has developed a low-boom supersonic configuration as shown in Fig. 1.

A vast knowledge base of analytical, computational, wind tunnel and flight data exists on the ASE subsonic vehicles and supersonic fighter aircraft, but considerably less data are available in this area for supersonic cruise configurations. Reference 1 describes the developments to date in ASE that are also utilized for this overall APSE effort. For propulsion system dynamics modeling and testing, there is not much relevant information in the open literature, ${ }^{2,3,5}$ besides the models that have already been developed to date for this APSE effort. Thus, the approaches and techniques utilized for modeling propulsion system component dynamics to integrate with the ASE system are mostly those developed in this effort.

The approach that has been adapted for the propulsion system modeling effort under APSE is to develop propulsion system component models that capture their dynamics with sufficient accuracy in the frequency range exhibited by the vehicle ASE dynamics and aerodynamics. The reason for this is that the propulsion system dynamics may sufficiently excite the ASE vehicle modes (via thrust force variations applied to the structure), leading to undesirable unsteady vehicle performance. The coupling of this APSE system is via the thrust dynamics from propulsion to ASE, and in turn the ASE system couples back to propulsion via the wing-structure displacements that perturb the flow field in front of the propulsion system. Therefore, the coupling of these systems effectively produces a closed-loop dynamic system, whose frequency range needs to cover the dominant modes exhibited by the vehicle ASE dynamics and aerodynamics.

\footnotetext{
${ }^{1}$ Senior Controls Engineer, Communications Instrumentation and Controls Division, 21000 Brookpark Rd/77-1, AIAA Member.

${ }^{2}$ Aerospace Engineer, Communications Instrumentation and Controls Division, 21000 Brookpark Rd/77-1, AIAA Member.

${ }^{3}$ Senior Aerospace Engineer, Aeropropulsion Division, 21000 Brookpark Rd/77-1, AIAA Member.
} 


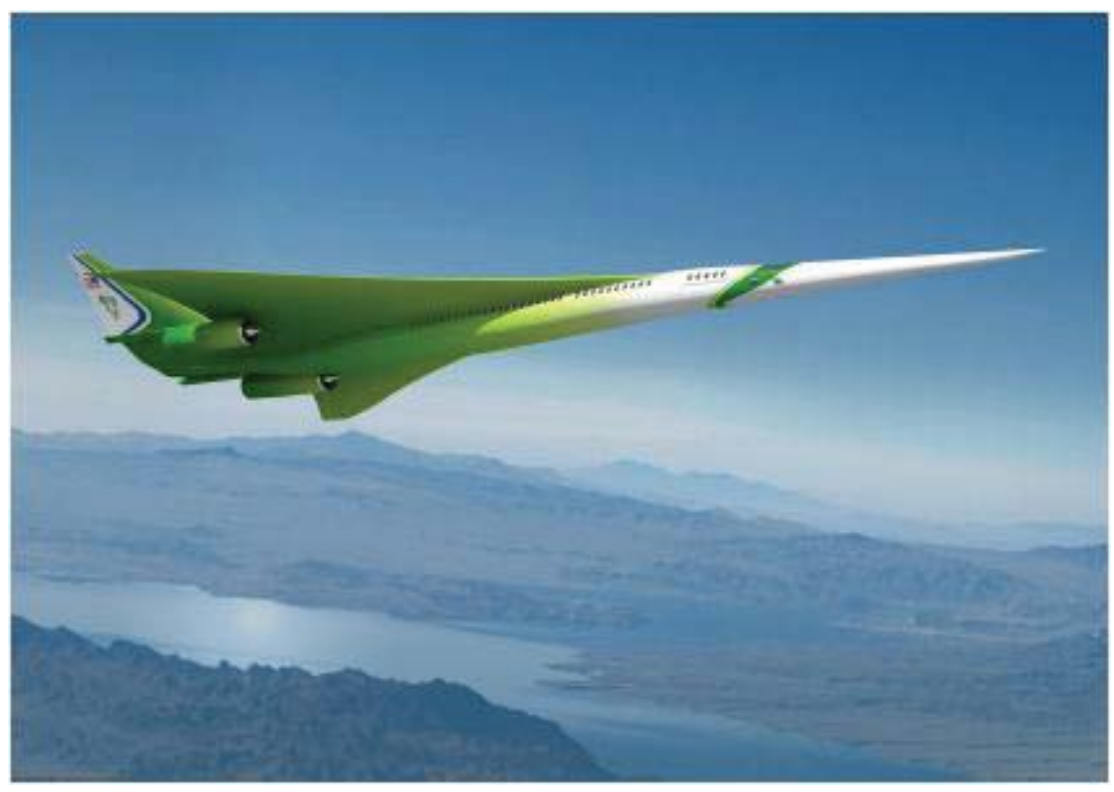

Figure 1. Artist's Concept of the Lockheed-Martin N+2 Configuration

These ASE frequencies can extend up to $60 \mathrm{~Hz}$ or more. The ASE frequency modes depend on the stiffness and mass of the vehicle, which varies for different types of vehicles ${ }^{4}$. Also, the frequency range depends on how many structural modes are included in the analysis (about the first 30 modes are typically included). Therefore, there is no set frequency range, per se for ASE modes. Nevertheless, provided an estimate for this frequency range (based on the number of ASE modes to be included), the propulsion system dynamics need to appropriately cover this range in order to develop appropriate APSE closed-loop system models. Moreover, since the poles and zeroes of the dynamic system influence the phase of the frequency response down to one decade below the actual pole-zero frequencies of these modes, the frequency range of the propulsion system modeling potentially needs to include dynamics up to a frequency decade above the expected ASE frequency range. The reason the phase of the dynamics becomes important in this type of system is because both the magnitude and phase of the dynamics are equally important in order to capture the stability behavior or the unsteady response of closed-loop systems.

In order to develop a dynamic propulsion system model to cover this frequency range, the component models also need to include the gas dynamics for the conservation of mass, momentum, and energy. Normally, the propulsion system dynamics modeling for this APSE effort needs to capture the 1-Dimensional (1D) axial flow, either in lump volume or in stage-by-stage form. There are some exceptions to this rule as follows. For the effect of flow distortion on the propulsion system dynamics, two-dimensional (2D) or parallel flow path modeling needs to be developed. Also, when mixing flow regions exist in a particular component design, like in the case of the $\mathrm{N}+2$ spike nozzle, 2D flow effects may need to be included. Similarly, the flow field may be inherently $2 \mathrm{D}$ or even three-dimensional (3D); this is the case with the axisymmetric $\mathrm{N}+2$ external compression inlet concept. In the latter case, the external flow field is conical, which is inherently $2 \mathrm{D}$, or $3 \mathrm{D}$ when pitch, yaw and roll disturbances are included. Propulsion system flow distortion can be caused by 2D or 3D flow disturbances due to structural mode excitations or vehicle maneuvers. The effects of flow distortion on APSE performance may be important to analyze, since even a small amount of flow distortion can have a significant effect on the propulsion system performance.

The paper summarizes the progress to-date to develop the APSE propulsion system models to satisfy the objective of developing an integrated dynamic model to conduct appropriate unsteady analysis of supersonic vehicle performance.

The paper is organized as follows. First, the modeling approach and component models developed for each of the propulsion system components will be described. This is followed by atmospheric turbulence modeling, and then followed by the overall propulsion system model developed to date and integrated with the ASE/aerodynamic model to create the first preliminary APSE model. Finally, some concluding remarks will be offered.

\section{Propulsion System Component Modeling}

This section will cover the various component models that comprise the overall propulsion system for the $\mathrm{N}+2$ concept vehicle, suitable for an integrated APSE system. Some of these propulsion system component models include more than one model variation. The purpose of these component model variations and how they fit into the overall objective of developing an APSE model to conduct performance studies will also be covered in this section.

A version of the $\mathrm{N}+2$ propulsion system component diagram and associated flows is shown in Fig. 2, which depicts a Variable Cycle Engine (VCE), with three flow paths; one core path and two bypass flow paths. In this figure HPC, HPT, LPT 
stand for the high pressure compressor, the high pressure turbine, and the low pressure turbine, respectively. The concept behind a VCE (an extension of a high bypass turbofan engine) is to optimize the cycle throughout the flight regime. In summary, this is accomplished by trimming the flow through the VCEFan stream so that the overall engine flow demand matches that of the inlet capture air in order to maximize pressure recovery and minimize flow spill, which adds to drag. The VCE flow stream begins with flow extracted from the fan tip region. This VCEFan stream is also utilized for noise abatement for supersonic overland flight. The control schedules required to optimize this VCE cycle are more complex compared to high bypass engines. The VCE cycle optimization requires altitude or speed scheduling of three Variable Guide Vanes, scheduling of the multi-stream nozzle areas (A8s), and scheduling of the inlet centerbody geometry.

The VCE operation to optimize the cycle can be summarized as follows. Given a corrected fan speed schedule corresponding to altitude and Mach number and inlet captured mass flowrate, this sets the engine face boundary conditions. Also, given the corresponding choked nozzle area (A8s) schedules, this provides the flow demand for the engine. The difference between the captured mass flowrate of the inlet and the flow demand of the core plus the main bypass nozzle(s) is passed through the VCEFan flow stream, whose nozzle is not choked. This is done by also utilizing a Variable Guide Vane (VGV) control schedule for the VCEFan. The rest of the VGVs control schedules for the fan and HPC are used to enforce operation of the compression systems on a desired operating line of their respective Pressure Ratio (PR) vs. corrected mass flowrate maps. For the common plenum nozzle VCE version shown in Fig. 2, the nozzle choking sets the overall flow demand for the main bypass and the core, while the LPT choking sets the flow demand of the core. The difference between those flow demands is forced through the bypass. The bleed flows are governed by the differences in choking between the core components and the bleed extraction flow areas.

\subsection{Compressor Modeling}

The N+2 VCE engine is a dual spool engine. The incoming flow splits between the fan and the VCEFan. The flow splits again before it reaches the HPC in the core path. The external VCE flow path, the Fan, and HPC all have variable geometries by utilizing separate VGVs. In this effort, a compressor has been generically modeled in 3 different ways. First, by utilizing lump volume dynamics and component characteristics (maps) based on the approach started in Ref. 2 and improved in Ref. 5 and 6. Second, by utilizing a stage-by-stage modeling approach developed in this effort for higher dynamic fidelity. Third, by developing an approach that starts with stage-by-stage modeling and then implements 2D parallel flow path modeling to model both axial and rotational flows in the compressor stages.

The parallel flow path component models have been developed to address the effects of flow distortion on the propulsion system and APSE performance. Lump volume dynamics means that the particular component model is represented by a single

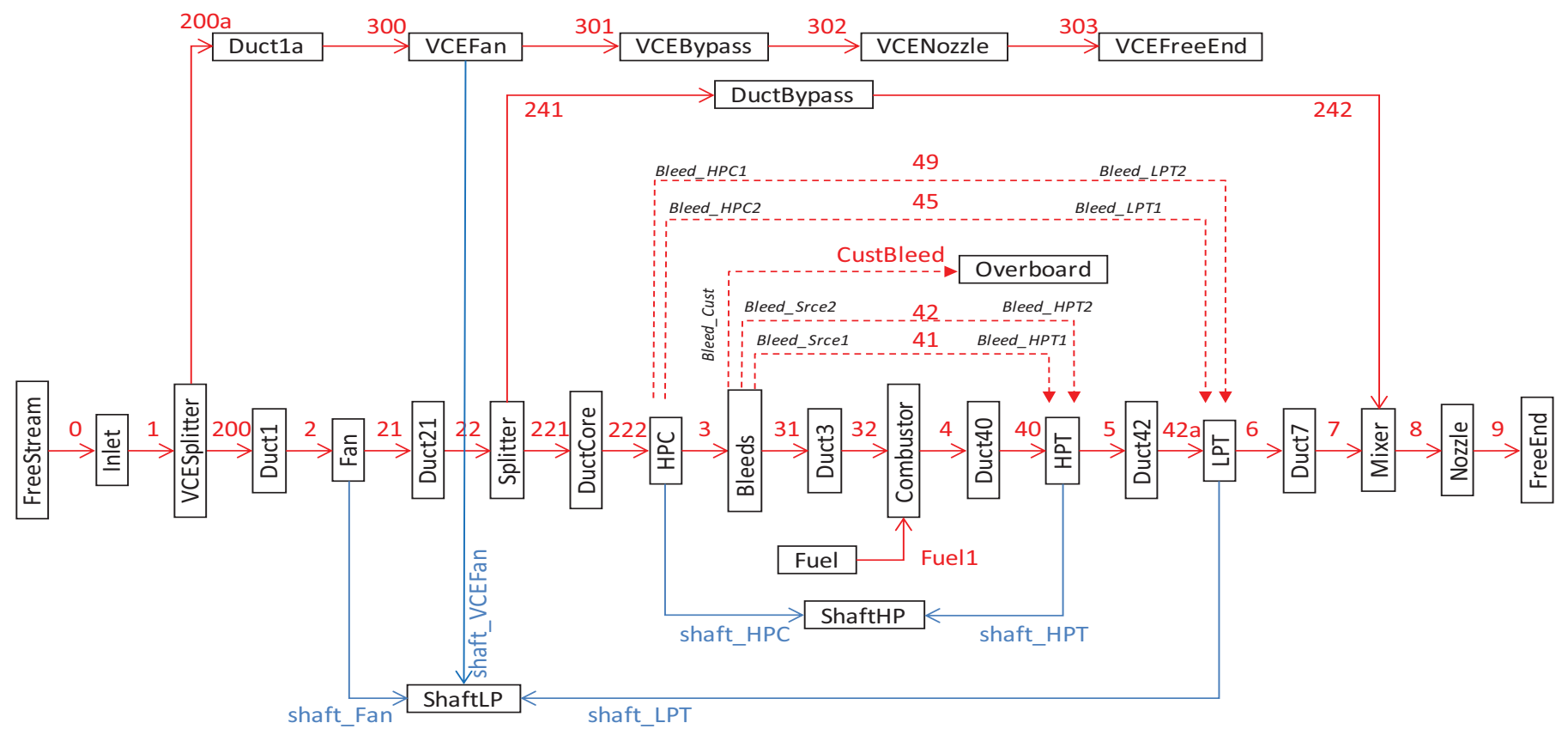

Figure 2. VCE propulsion system component diagram and associated flow paths. Flow paths are shown in red color and shaft component connections are shown in blue. 
volume with associated performance characteristics and with the conservation dynamics of continuity, momentum, and energy. This is compared to stage-by-stage component modeling where each stator-rotor stage is separately modeled as a dynamic volume. Lately, the VGVs were also included in the respective VCE component models in order to model the variable geometry of these components and to derive control schedules to operate the compressors and the VCE appropriately throughout the operating regime. These modeling approaches are summarized in the following subsections.

\subsubsection{Compressor Volume Dynamics Model}

The N+2 VCE propulsion system model presently consists of lump volume dynamic models of the various engine components including the ducts as seen in Fig. 2 (i.e., for all the engine components except for the inlet and nozzle). The approach utilized is the same as that covered in Ref. 2 and 5 using the same MATLAB/SIMULINK ${ }^{\circledR}$ component blocks that have been originally developed. The differences are the component characteristics and the associated geometry properties, which are specific to the particular engine/component design. These performance maps and the component geometries as well as the initial conditions for initializing the component states of the VCE model have been mostly imported from the Numerical Propulsion System Simulation (NPSS), which has been utilized so far to develop the N+2 cycle design.

\subsubsection{Variable Guide Vane Model}

The VGV model is developed and integrated with the compressor models to allow for variable compressor geometries necessary to operate the VCE within its operating range of power and altitude, while forcing the compressor (via control schedules) to operate on a desired operating line of the compressor pressure ratio (PR) vs. corrected mass flowrate (CMF) performance map. The VGV's are used for the LPC, the HPC, and the VCEFan in order to properly split the flows to optimize VCE operation.

The VGV model consists of performance characteristics with sets of maps at different VGV angles, which are interpolated. Additionally, for this dynamic model the effective cross section area is calculated as a function of IGV angle. The maps supplied by NPSS include the overall incidence angle. The incidence angle, however, is taken out in this model and the reference angle with no VGV turning is referred to as the $0^{\circ}$ angle. This is done in order to implement a reference cross sectional area for the volume dynamics equations ${ }^{5,6}$ that corresponds to $0^{\circ} \mathrm{VGV}$ turning angle. For this VGV model, the mass flow parameter (MFP) is utilized and the compressor effective flow area is calculated as a function of the $\mathrm{MFP}^{7}$ and additionally, by taking into account here the cosine of the VGV angle as

$$
\begin{gathered}
A=\frac{\dot{W} \sqrt{T_{t}}}{M F P * P_{t} \cos (\alpha)} \\
M F P=M \sqrt{\frac{\gamma g}{R}}\left(1+\frac{\gamma-1}{2} M^{2}\right)^{\frac{\gamma+1}{2(1-\gamma)}}
\end{gathered}
$$

where A, MFP, $\dot{W}, P_{t}, T_{t}, \cos (\alpha), M$ are the component cross section area, the mass flow parameter, the component total pressure and temperature, the cosine of the VGV angle, and the component Mach number, respectively. The angle $\alpha$ is the VGV turning angle, itself (i.e. without including the overall component incidence angle). The variables $g$, $R$, and $\gamma$ stand for the gravitational acceleration, the universal gas constant, and the ratio of specific heats, respectively.

\subsubsection{Stage-by-Stage Compressor Model}

In addition to the lump volume compressor model, a technique was developed to convert the lump volume model into stageby-stage. Stage-by-stage compressor modeling (SBSCM) is important for a couple of reasons. First, the SBSCM model is necessary to develop parallel flow path models to study the effects of flow distortion on the propulsion system dynamics and for studying the compressor surge/stall dynamics. The reason for this is that flow distortion, which cannot be represented by a lump volume model, affects the dynamics of surge and stall. Second, the SBSCM provides more dynamic accuracy in the propulsion system model for APSE performance assessments. For instance, wave reflection could be an issue, which can affect the frequency response and the accuracy of the dynamic model. This accuracy can start to be impacted when the excitation frequency wavelength exceeds $1 / 4^{\text {th }}$ of the acoustic wavelength across the length of the device. If, for example, the compressor minimum Mach number is 0.3 at cruise (speed of sound is $\approx 295 \mathrm{~m} / \mathrm{s}$ ) and if the compressor model needs to have dynamic accuracy up to $100 \mathrm{~Hz}$, then the dynamic accuracy of the model can start to deteriorate if the compressor distributed length exceeds $1 / 4 *(0.3 * 295 / 100)=0.22 \mathrm{~m}$.

If a dynamic compressor SBS model is being developed for an existing engine and if information on the engine's respective geometries and associated performance exists, the use of this information would be the most expedient way to develop such a 
model. Most often, however, this is not an option, especially with new conceptual engine designs. Thus, the steps of developing an SBS model, starting from a lump volume compressor model, are summarized here.

First, one needs to know or decide the number of stages $(J)$ that will be incorporated into the compressor design. Then the compressor pressure ratio (PR) vs. corrected mass flowrate $\left(\dot{W}_{c m f}\right)$ and the efficiency $(\eta)$ vs. PR maps ${ }^{5}$ of the lump volume component model can be scaled for each of the stages as follows:

$$
P R_{s t}=\sqrt[J]{P R_{l m p}}, \quad \eta_{s t}=\sqrt[J]{\eta_{l m p}}
$$

where the subscripts $\operatorname{lmp}$ and $s t$ stand for lump and stage, respectively. If no specific information exists, the lengths of all stages can be assumed the same, which would be equal to the length of the lump compressor divided by $J$. Initially, in the absence of a VGV model, the lump compressor bleed rate was utilized to devise a control schedule to force the compressor to operate on a desired operating line on its PR map. For the SBSCM, this bleed was fictitiously incorporated in each stage and set equal to the lump compressor bleed divided by $J$. The compressor operating line is normally chosen to be situated near the knees of the speed lines, cutting across the maximum efficiency lines, as shown in Fig. 3. Alternatively, the operating line can be designed to maintain a minimum stall margin. The operating line on the PR map for an early design, based on the derived control schedule using the bleed, is shown in Fig. 3.

When starting with a lump volume compressor model and directly proceeding to develop a SBSCM, the simulation normally will go unstable. The reason is that the initial state conditions at an operating point (let's say $100 \%$ speed at cruise), extracted from the lump volume model would be too far off the SBSCM actual operating conditions. Therefore, the scaling needs to be done in steps (ex., full scale to $3 / 4$ scale, to $1 / 2,1 / 3,1 / 4, . ., 1 / 8$ and so on, until the final stage size of the SBSCM is developed).

In each scaling step, the isolated compressor simulation is run to steady state and the final state conditions are stored and substituted for the initial conditions to initiate the next simulation run. This process is repeated until the starting transient sufficiently subsides.

When the final size of the compressor stage model is successfully developed, the next step would be to stack together a $J$ number of these stage models to obtain an overall SBSCM. However, doing so in one-step will not work. Since the Mach number drops across the compressor (from upstream to downstream), each of the stages ( $i$ ) must have a unique cross section area and therefore, a unique geometry. So knowing or estimating (from the lump volume simulation) the compressor input Mach number $\left(M_{i n}\right)$, and the Mach number drop across the whole compressor component $(\Delta M)$, the Mach number $\left(M_{i}\right)$, for each successive stage can be approximated as

$$
M_{i}=M_{i n}-\frac{i}{J} \Delta M
$$

The next step will be to stack the stage models one at a time, starting with the stage that is further upstream. As will be detailed later, in each step the cross sectional area of the stage $i$ may be calculated using the equation

$$
A_{i}=\frac{\dot{W}_{c, i}}{\rho_{s v, i} M_{i} \sqrt{\gamma R T_{s, i}}}
$$

where $\dot{W}_{c, i}, \rho_{s v, i}$, and $T_{s, i}$ are the stage mass flowrate, static density, and static temperature, respectively. And the subscripts $c$ and $v$ stand for stage characteristics and stage volume, respectively ${ }^{5}$. The same modeling approach utilized for the lump volume compressor was also used for the SBSCM, but with the new maps, geometry, and initial conditions described here.

In the process of stacking the unique stage models one at a time, a procedure similar to the initial scaling process is followed, whereby the model simulation is run successively with the final conditions of one iteration substituted as initial conditions for the next iteration, until the initial transient subsides. Before the process with the initial conditions starts, however, the Mach number estimator that should be included as part of the compressor model ${ }^{5}$ (based on Eq. (5), but solving for $M$ instead of A) should be disconnected. Instead, the Mach number of the last

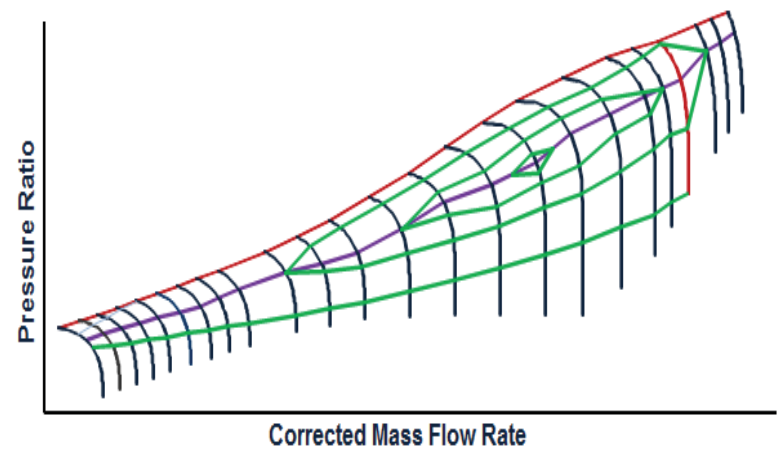

Figure 3. Compressor pressure ratio map showing the operating line in purple color and the efficiency islands in green. 
stage connected to the model should be set to the calculated stage Mach number of Eq. 4. This stage Mach number should be utilized until the step of working with the initial conditions is finalized. The reason for that is that a mismatch in the initial conditions will also cause Eq. (5) for the stage Mach number and the area to become mismatched, causing additional numerical instability problems. Thus, for each step, as the initial conditions are replaced by the final conditions, these conditions should be used to calculate a new cross section area for the last stage connected by utilizing Eq. (5). This process should be repeated until both the initial startup transient sufficiently subsides and Eq. (5) is sufficiently balanced. Once this process is completed, the Mach number estimator for this last stage should be reconnected, and the simulation run to verify the Mach number calculated for the last stage. Also, it should be verified that reconnecting the Mach number calculation block doesn't appreciably change the final conditions. This process is repeated for each successive stage until all the stages are successfully connected to develop an SBSCM.

This model should be able to operate near the desired overall compressor operating point that was used to develop the SBSCM. However, to operate the model at some other operating point, a control schedule would be needed - topic which will be covered in the next section.

\subsubsection{Compressor Control Schedules}

Compression system control schedules utilizing the VCE variable component geometries of the VGV's can also be derived utilizing the dynamic engine simulation test bed that is presented in this paper. This can be done through a process whereby the dynamic engine simulation is run successively at incremental operating conditions to generate initial conditions for the compressor and corresponding boundary conditions. Following, an isolated lump volume compressor model is developed for each VGV by isolating these models from the existing VCE simulation. A corresponding compressor plug nozzle simulation is also developed for each VGV by utilizing the compressible flow choked flow equation. ${ }^{5}$ A certain parameter that of this equation that pertains to the nozzle area is adjusted in order to produce the same mass flow conditions at the various operating points as those of the engine. Lastly, the corresponding VGV angle is adjusted to develop a control schedule to force the compressor to operate on the desired operating line on the compressor PR vs. correct mass flowrate map. The details of this compressor control schedule development process will be described in an accompanied NASA Technical Memorandum, which is in progress.

Because the process utilized with the dynamic engine model to determine compressor control schedules is rather laborious, instead, NPSS has recently been utilized. The dynamic VCE model will be utilized in this later process for verifying these control schedules.

\subsubsection{Compressor Parallel Flow Model}

The purpose of parallel flow path modeling is to develop higher fidelity models to assess the effects of flow distortion on the dynamics of surge/stall as well as the overall propulsion and APSE dynamics, as the propulsion system is sensitive to flow distortion. For distortion modeling and analysis, all the propulsion components in the core path of the engine upstream of the combustor need to be modeled in a similar way to the approach that will be covered here for the compressor.

The compressor parallel flow modeling approach implemented in this effort starts with an SBSCM, where each stage is subdivided to 2, 4, or more sub stages, as shown in the pictorial diagram of Fig. 4. In this figure the pairs of Ps and Ts stand for total pressure and temperature, respectively and they indicate a flow distortion pattern at the compressor inlet. The numbers that follow these variables indicate the sector of the subdivided compressor stage. Both axial and rotational flows are modeled in this approach for a $2 \mathrm{D}$ flow model. This pictorial diagram in Fig. 4 shows an 8 -stage compressor divided into 4 parallel paths $(8$ parallel paths usually would be the most required for sufficient distortion modeling). A mixing volume is included at the compressor outlet where all the flows come together before the flow enters the combustor, as shown in Fig. 4 . The different sets of pressures and temperatures seen at the compressor inlet shown in Fig. 4 can be adjusted to simulate a desired distortion pattern.

This model has been developed in cylindrical coordinates. Unlike what has been done in the past, ${ }^{8,9,10}$ it is deemed important in this effort to also include flow variations in the rotational direction since the incipiency of stall and surge starts from flow variations in that direction. The assumptions used to develop this model are that the flow is compressible, inviscid, and adiabatic. Similar to the engine volume component models described in Ref. 5, it is also assumed here that the convective momentum terms in the conservation equations are negligible and they can be omitted, and the same is assumed to be true for the body forces for the gravitational terms (due to relatively small physical dimensions).

Based on these assumptions, the following general expression was used to express this model for the conservation of continuity, momentum, and energy.

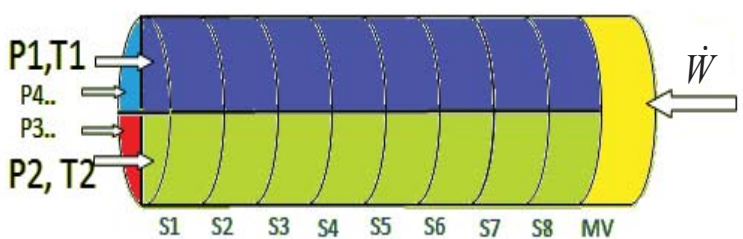

Figure 4. Pictorial diagram of a parallel flow 8-stage compressor model. 
Table 1 - Equation (6) term definitions.

\begin{tabular}{|c|c|c|c|c|c|c|}
\hline $\mathrm{j}$ & $\mathrm{Wj}$ & $\mathrm{Fxj}$ & $\mathrm{F} \phi \mathrm{j}$ & $\mathrm{Sj}$ & $\mathrm{a}_{\mathrm{xj}}$ & $\mathrm{a}_{\phi \mathrm{j}}$ \\
\hline 1 & $\rho_{s}$ & $\rho_{s} u$ & $\rho_{s} w$ & $\frac{1}{r}$ \\
\hline 2 & $\rho_{s} u$ & $\rho_{s} u$ & $\rho_{s} u$ & $-\frac{\partial P_{s}}{\partial x}$ & $u$ & $\frac{w}{r}$ \\
\hline 3 & $\rho_{s} w$ & $\rho_{s} w$ & $\rho_{s} w$ & $\frac{1}{r} \frac{\partial P_{s}}{\partial \varphi}$ & $u$ & $\frac{w}{r}$ \\
\hline 4 & $\frac{P_{s}}{\gamma-1}+\frac{\rho_{s} V^{2}}{2}$ & $\frac{\gamma P_{s} u}{\gamma-1}+\frac{\rho_{s} u^{3}}{2}$ & $\frac{\gamma P_{s} w}{\gamma-1}+\frac{\rho_{s} W^{3}}{2}$ & 0 & 1 & $\frac{1}{r}$ \\
\hline
\end{tabular}

With $W_{j}, F_{j}$, and $S_{j}$ respectively identifiable as the state terms, the flux terms, and the source terms, with the following definitions for these terms, as listed in Table 1 . The terms $u, w, \mathrm{r}, a_{x j}$, and $a_{\varphi j}$ are the axial flow velocity component, the rotational velocity component, the radius, the flux term coefficient, and the source term coefficient, respectively. Central difference was applied to Eq. (6) to approximate the spatial derivatives, with the compressor stages indexed in terms of $n, n=1,2 \ldots, k(k=$ number of compressor stages) and with the compressor parallel segments indexed as $m, m=1,2 . ., q$ ( $q=$ number of parallel compressor segments), which results in the following expression:

$$
\frac{\partial}{\partial t}\left(W_{j, n, m}\right)=-a_{x j, n, m}\left(\frac{F_{x j, n+1, m}-F_{x j, n, m}}{\Delta x}\right)-a_{\varphi j, n, m}\left(\frac{F_{\varphi j, n, m+1}-F_{\varphi j, n, m-1}}{2 \Delta \varphi}\right)+\frac{S_{j, n, m-1}-S_{j, n, m+1}}{2 \Delta s}
$$

To develop these formulations, it is assumed that the length of the compressor stages $(\Delta x)$, and the angular variations $(\Delta \varphi=$ $\left.\frac{2 \pi}{q} \mathrm{rad}\right)$, are all the same, where $\Delta s$ is either equal to $\Delta x$ or equal to $\Delta \varphi$ depending on the partial expression for the respective terms in Table 1.

As mention before, the mixing volume is appropriate when the combustor is the next downstream component, where it can be assumed that flow distortion can be neglected beyond this point due to the combustion mixing and reactions processes. For parallel flow path modeling of the inlet, the fan, and ducts upstream of the HPC, a mixing volume similar to the one used for the HPC can also be utilized to isolate the component parallel flow path models. However, these mixing volumes would be removed when these components are interfaced together. The mixing volume in Fig. 4 utilizes volume dynamics as covered in Refs. 2 and 5 , except that these dynamics were modified to include the appropriate mixing flows. As such, the gas dynamics for the mixing volume by applying conservation of continuity, momentum, and energy are

$$
\begin{gathered}
\frac{d}{d t} \rho_{s, m v}=\frac{1}{V_{m v}}\left(\dot{W}_{m v}-\dot{W}_{c b}\right) \\
\frac{d}{d t} \dot{W}_{m v}=\frac{A_{m v} g}{l_{m v}}\left[\sum_{m=1}^{q}\left(\beta_{m} P_{t m, n=k}\right)-P_{t, m v}\right]\left(1+\frac{\gamma_{c p}-1}{2} M_{m v}^{2}\right)^{\frac{-\gamma_{c p}}{\gamma_{c p}-1}} \\
\frac{d}{d t}\left(\rho_{s, m v} T_{t, m v}\right)=\frac{\gamma_{m v}}{V_{m v}}\left[\dot{W}_{m v} \sum_{m=1}^{q}\left(\beta_{m}^{2} T_{t m, n=k}\right)-\dot{W}_{c b} T_{t, m v}\right]
\end{gathered}
$$

where $A, l$, and $V$ are respectively the area, length, and volume of the mixing volume. The subscripts $m v, c p$, and $c b$ in this case stand for mixing volume, compressor, and combustor, respectively. The parameter $\beta$ is defined as the weighting factor of the angular coverage of the specific parallel compressor segment divided by the total angular coverage of the compressor, or $360^{\circ}$. In Fig. 4, all four segments have equal angular coverage (i.e., $\left.\beta_{m(m=1 \text { to } 4)}=1 / 4\right)$. The length of the mixing volume was chosen to be comparable to the length of a stage. This was done in order to avoid the possibility that the compressor dynamics would be 
dominated by the mixing volume (if the mixing volume size is large compared to that of the stages), and to avoid high frequency dynamics if the mixing volume size is too small, which would unnecessarily slow down the simulation.

The mass flowrates coming from the mixing volume as inputs to each parallel flow path of stage $n$, can be computed as

$$
\dot{W}_{m, n=k}=\beta_{m} \dot{W}_{m v}
$$

If any of the stages includes bleed flow, then this bleed flow needs to be accounted for in the respective flux losses in Eq. (6), whereby the bleed can also be distributed to the particular stage parallel elements by the coverage factor, $\beta_{m}$. Alternatively, the entire bleed could be attributed to single parallel flow element when appropriate. The compressor PR maps from the SBSCM are also utilized for this model. However, the PR map for each parallel stage element is modified by scaling the corresponding stage corrected mass flowrate (CMF) by its coverage factor, $\beta_{m}$.

Figure 5 shows a generic PR map of the compressor used for this simulation that includes normal operation, rotating stall, and back flow regions. The rotating stall and back flow regions are required for successfully modeling stall dynamics. Figure 6 shows the operation of the $7^{\text {th }}$ compressor stage for a pressure distortion less than $1 \%$ applied to path 1 . This figure shows (in a snapshot) parts of the stage operating in the rotating stall region. This shows that these undesirable events initially start with initially parts of the stage moving into the surge region before the whole stage surges or before the whole compressor moves into surge. Thus, the dynamics that describe the onset of surge are best captured with 2D type flow path modeling. Figure 7 shows the rotational velocities during a stall. As seen in this figure, the gas dynamics of stall are relatively fast and cannot be captured by reduced order simulations.

\subsection{Combustor Modeling}

The VCE combustor has been modeled with lump volume dynamics and performance characteristics, including fuel dynamics, fuel/air ratio for lean combustion, and the associated fuel type enthalpy as described in Ref. 2 and 5. The reader is referred to these references especially Ref. 5, for more details.

\subsection{Turbine Modeling}

The turbine modeling for the VCE also follows the same modeling approach outlined in Refs. 2 and 5 (especially Ref. 5), which is based on performance characteristics and lump volume dynamics. For the VCE, there are 2 spools as mentioned earlier and both a HPT and a LPT have been modeled with the appropriate cooling flows originating from the compressor as seen in Fig. 2. Separately, a stage-by-stage turbine modeling (SBSTM) approach has also been developed for the turbine, which has not yet been incorporated into the VCE simulation.

\subsubsection{Stage-by-Stage Turbine Model}

The SBSTM approach utilized is about the same as that described for the compressor (SBSCM). For the turbine, more care should be exercised for generic maps to not push the operation of individual stages or the overall turbine operation too deep into the choked region. That's because at some point deep into the choked region, the simulation will become unstable, which is similar to actual turbine operation. The reason is that the deeper the turbine operates into the choked region the higher the delta

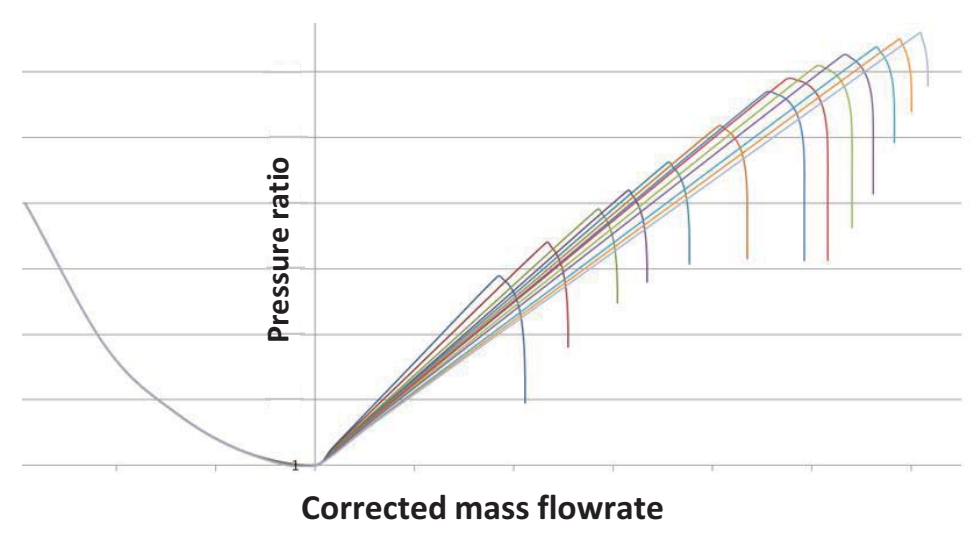

Figure 5. Generic PR map utilized for compressor parallel flow path modeling, showing speed lines having their own surge/stall line.

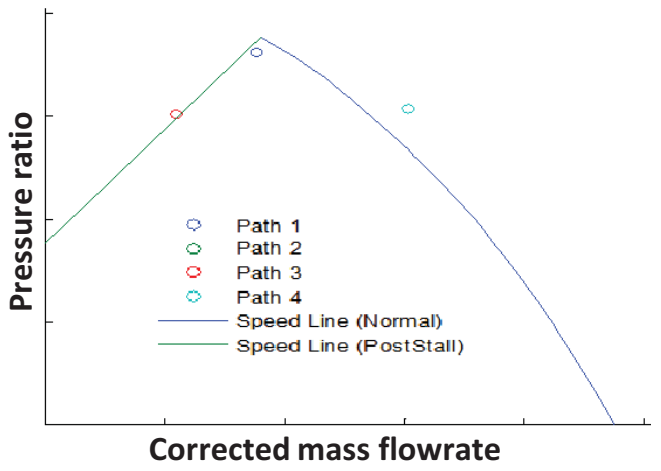

Figure 6. PR speed line map for $100 \%$ throttle, showing in a snap shot part of compressor stage 7 in the rotating stall region due to the distortion pattern applied. 
pressure ratio would be for a small deviation in the corrected mass flowrate (this also holds true for the compressor operation).

\subsection{Afterburner}

The VCE is envisioned to incorporate a small afterburner downstream to help provide more thrust during take-off and acceleration to cruise. Currently, the VCE model does not include an afterburner. In the future, the afterburner model is envisioned to be similar to that covered in Ref. 5.

\subsection{Rotor Models}

The VCE is a two-spool engine, and as such, two rotor models are employed in this engine. One of the rotors ties the fan with the LPT and the other ties the HPC to the HPT. Except for the specific extracted flows, these models are basically the same as the rotor model covered in Ref. 2 and Ref 5. In Ref. 6 the rotor models account for VCE specific cooling and other extraction flows. Recently, the model described in Ref. 6 has been updated to include all the cooling and extraction flows shown in Fig. 2.

\subsection{Duct Models}

As seen in Fig. 2, there are various ducts included as part of the VCE design architecture. These ducts have also been modeled based on lump volume dynamics, as the gas dynamics of their respective volumes cannot be ignored for the overall propulsion system dynamics for APSE. For distortion modeling and analysis, the ducts situated upstream of the HPC would also need to be modeled utilizing the parallel flow path modeling approach discussed in section 2.1.5. However, unlike the compressor parallel flow path modeling, no performance maps will be used in this case. Also, a duct would be modeled as a lump volume or even as a multistage (distributed volumes), depending on the acoustic length or the length of the particular duct compared to the compressor stages.

\subsection{Inlet and Nozzle models}

Initially for the engine in Ref. 5 and for the VCE in Ref 6, the inlet was modeled simply for the temperature and pressure rise across the component, without including any dynamics. For the nozzle, the compressible flow choked flow equation ${ }^{2,5}$ was utilized together with the nozzle lump volume to model this component. However, it was deemed important from the beginning to model both of these devices utilizing CFD methods in order to capture their respective dynamics due to the formation of shocks, which potentially could significantly influence the dynamics of these components. To model these devices for steady state performance accuracy utilizing traditional CFD methods can prohibitively increase the simulation complexity and execution time for a propulsion system model. However, it was envisioned early on that for sufficient dynamic accuracy in the frequency range of interest for APSE closed-loop stability, the grid resolution for the inlet and the nozzle could potentially be reduced by several orders of magnitude.

\subsubsection{Inlet Model}

For the inlet, a code named LArge Perturbation INlet (LAPIN) ${ }^{11}$ based on a CFD method of characteristics, was developed years ago at NASA GRC. This code was initially utilized in this effort, but abandoned later. This is because the code was found to be difficult to modify (due to its complexity). Also, this code did not permit required radial changes to the centerbody contour of axisymmetric inlets. Because of these difficulties, a quasi-1D CFD mixed compression inlet code named NOIMA ${ }^{12}$ (NOnlinear Inlet Modeling Assembly) was developed. The VCE concept inlet, however, is an external compression axisymmetric type, which is more difficult to model, especially without the availability of test data. Subsequently, a test was conducted at NASA GRC for a scaled version of the same type of inlet, and test data were utilized to develop such a quasi-1D external compression inlet model ${ }^{13}$.

This external compression axisymmetric inlet $\operatorname{model}^{13}$ was implemented to include a compressible flow oblique shock CFD domain with flow propagation time delay dynamics, followed by a quasi 1D CFD domain between the normal shock and the cowl that includes leakage fluxes, followed by an internal inlet quasi 1D domain. The first two are moving computational domains, whose boundaries are dependent on the position of the external shock. An actuator model was included to allow for variable inlet geometry. This modeling methodology was recently extended to model the concept VCE inlet. For inlet-engine integration, the inlet-engine interface boundary conditions were changed from the typical fixed pressure boundary condition to specification of the mass flowrate demanded by the engine and with total pressure and temperature as outputs. To make this 
change to the boundary conditions, an appropriate size constant area volume dynamics cell was introduced at the inlet-engine interface. The engine was then successfully integrated with this inlet model.

Simulation results from the integrated inlet-VCE model are shown in Fig. 8 and Fig. 9. Figure 8, on the left, shows the engine normalized thrust starting with the engine by itself, and then for the integrated inlet-engine as the two systems are switched to run together at $2.0 \mathrm{sec}$. On the right, it shows the normalized mass flowrate at the inlet exit (before the constant area exit cell) starting with the inlet by itself (inlet starts at $1.8 \mathrm{sec}$ ), and then for the integrated inlet-engine as the 2 systems are switched to run together at $2.0 \mathrm{sec}$. Figure 9, on the left, shows the fan speed response for steps in the engine speed command and the corresponding pressure response at the engine face on the right. The behavior is expected, except for the sustained oscillation seen, which is due to coupling between the dynamics of the speed feedback control loop and the inlet. This is expected to be corrected in the future with improved control designs.

In a parallel effort under this task, the inlet was also modeled using more traditional CFD methods, but with the emphasis on capturing the dynamics of the inlet and analyzing the grid resolution required for dynamic accuracy. The criteria developed for sufficient dynamic accuracy are covered in the next subsection. Because the inlet is axisymmetric, the flow field externally is conical and inherently 3D when vehicle maneuvering is also accounted for. Therefore, in this effort, the truth inlet model for the VCE concept inlet was developed in 3D with high grid resolution. Two dimensional inlet models were also developed with the objective of utilizing these higher fidelity models to develop dynamic correction factors in order to allow a quasi 1D model that adequately describes the inlet dynamics to be utilized for the VCE propulsion system. However, for flow distortion analysis of the propulsion and APSE systems, it would still be necessary to use versions of these high fidelity models or a parallel flow model. Figure 10 shows a 2D simulated pressure flow field for the $\mathrm{N}+2$ inlet simulation that was developed under this effort for the purpose of capturing the inlet dynamics in the frequency domain. Figure 11 shows the average axial static pressure distribution across the length of the inlet that was extracted from the $2 \mathrm{D}$ simulation. The pressure distribution from this 2D simulation is comparable to the results obtained from the quasi 1D simulation of the VCE inlet, which is shown plotted against the inlet geometry in Fig.12. The differences in pressure are mainly due to the external compression

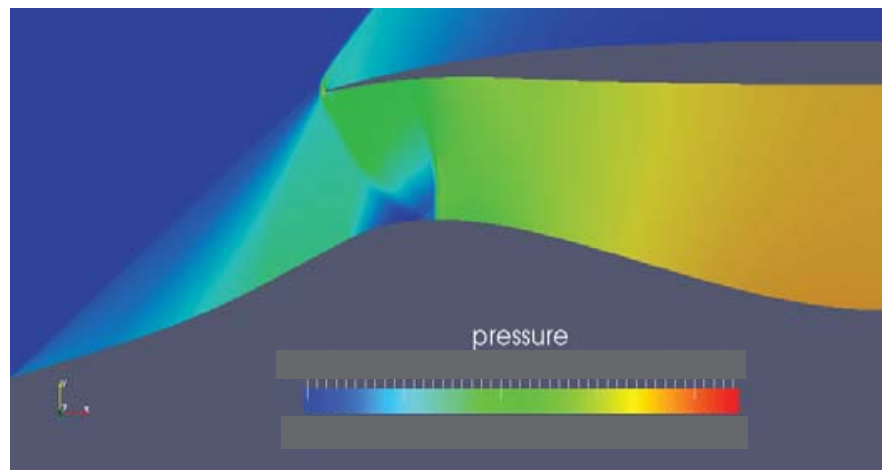

Figure 10. Pressure distribution of the 2D dynamic Inlet Model.
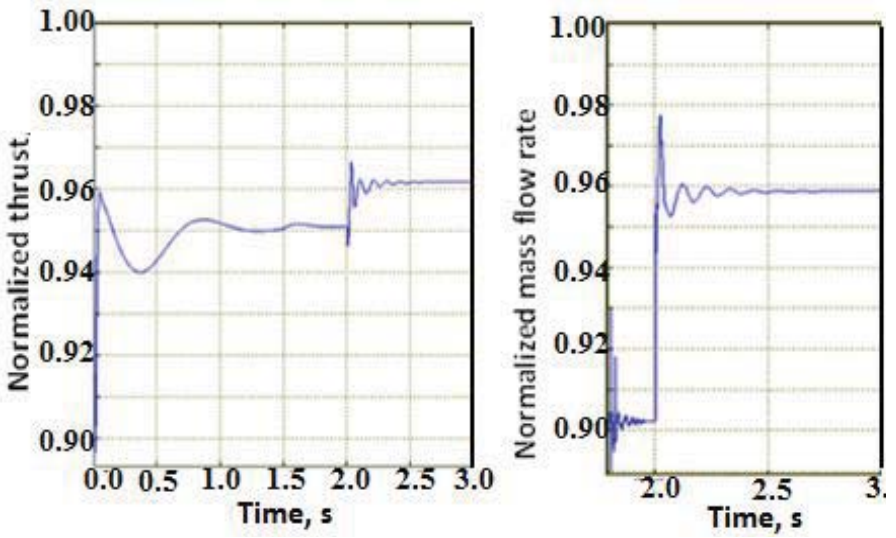

Figure 8. Engine thrust and mass florate for the integrated inlet-engine simulation.
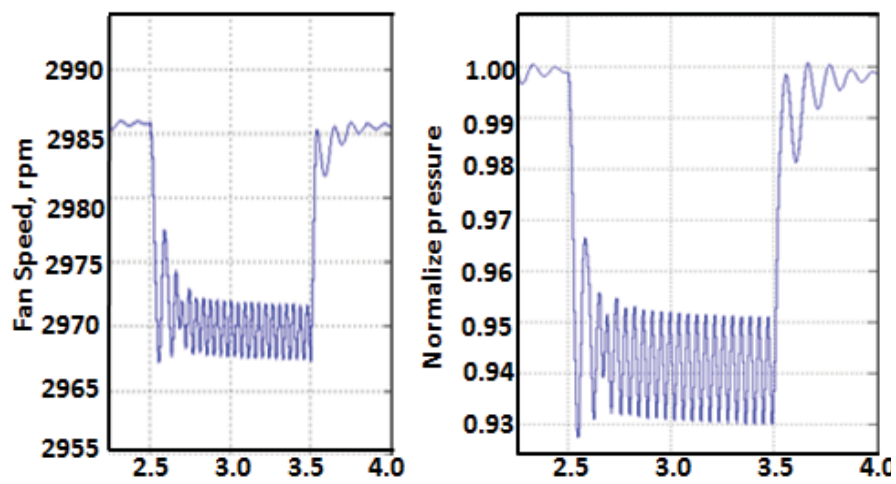

Figure 9. Fan speed end engine face pressure response of the integrated engine-inlet due to steps in fan speed command. model in the 1D simulation, which at present incorporates an oblique shock train for a rectangular inlet rather than a conical shock and Mach waves for axisymmetric type inlets. Also, due to the viscosity differences (artificial viscosity is used for the simulation in Fig. 12), the normal shock is smeared for the 1D simulation. However, the objective here is not to precisely match the steady state results, but rather to achieve sufficient dynamic accuracy. That is, the objective is for the 1D simulation to exhibit the dynamics of the respective 2D and 3D simulations in the frequency domain. For instance, the differences in $\mathrm{dB}$ magnitude of the steady state simulation for a frequency domain response comparing the $1 \mathrm{D}$ with the $2 \mathrm{D}$ simulation of the pressure distribution is not too appreciable (about $3 \mathrm{~dB}$ at most). A complete comparison of the dynamic responses in the frequency domain is expected to be covered 


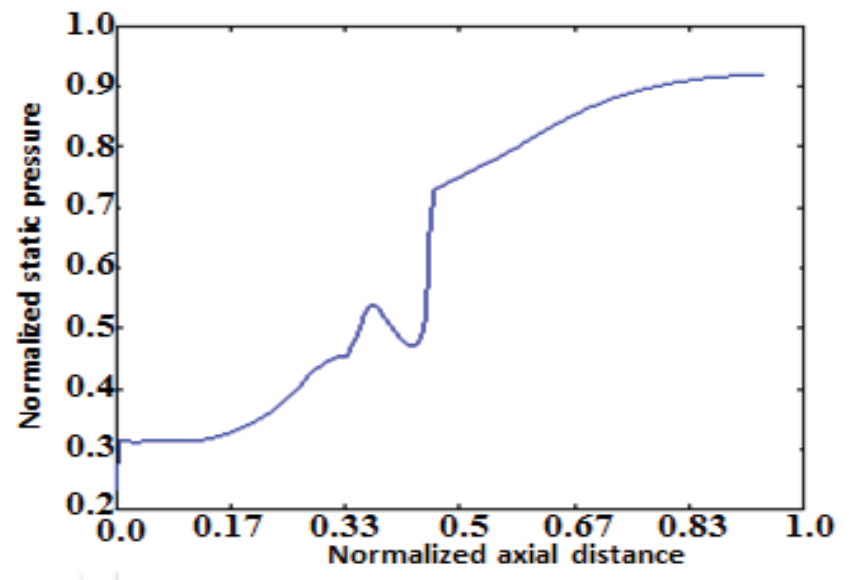

Figure 11. Normalized pressure distribution across the length of the inlet from the 2D simulation.

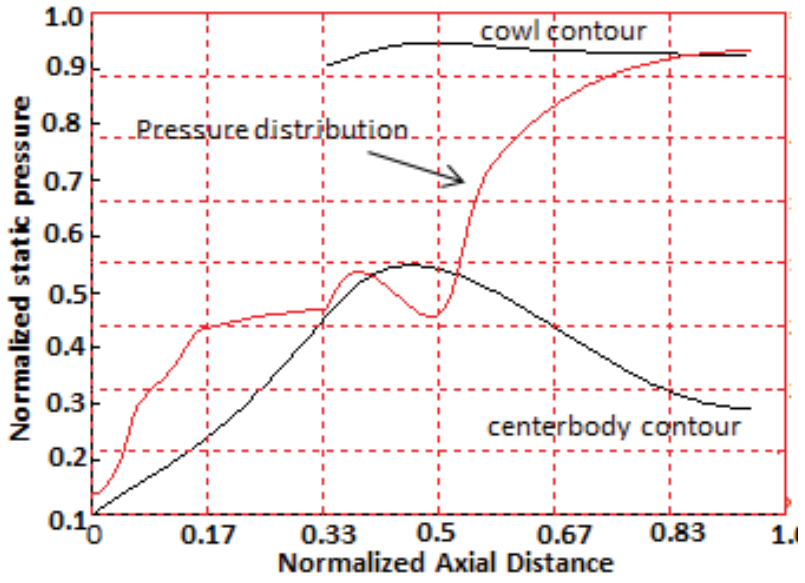

Figure 12. Normalize pressure distribution across the length of the inlet from the quasi-1D simulation

in a future report.

\subsubsection{Nozzle Model}

As mentioned before, the nozzle was initially modeled utilizing the compressible flow choked flow relationship. ${ }^{2,5}$ Later, a generic 1D Convergent-Divergent (CD) nozzle was also developed utilizing the McCormick method. ${ }^{14}$ The concept $\mathrm{N}+2 \mathrm{VCE}$ nozzle is a multi-flow path CD type nozzle with an external spike. The internal flows come together externally, where some mixing of the flows takes place and the combined flow expands along the length of the spike. From preliminary results, the internal duct dynamics can be sufficiently described utilizing $1 \mathrm{D}$ CFD modeling approaches. ${ }^{15}$ Results that are still in progress, comparing frequency responses of the internal ducts (1D) with that of the entire inlet (1D internal and 2D external), indicate that the nozzle dynamics in the desired frequency range can be sufficiently described by only simulating the internal 1D ducts of the nozzle.

As discussed earlier, it is important to capture the dynamics of the propulsion system in the range of the expected ASE dynamics in order to develop a dynamically comparable APSE system model to conduct related integrated vehicle performance studies. Towards this objective, the nozzle is also a key component whose dynamics need to be properly modeled. So based on that and as discussed earlier, it has been determined that the nozzle dynamics need to be accurately represented in the range of up to about $600 \mathrm{~Hz}$. A criterion was also developed to determine the grid resolution required to provide for sufficient dynamic accuracy. As such, the criterion for a sufficient grid resolution in this study was determined to be a maximum of about 3 dB magnitude deviation from the truth or the high-resolution CFD model, with this deviation to be in the form of an offset in the frequency range of interest. By specifying such a fixed offset, the frequency modes of the system are preserved throughout the frequency range of interest. The phase accuracy criterion was specified to be within a few degrees (less than 10 degrees). Magnitude and phase accuracy criteria are required to provide a deterministic means of capturing the dynamic response (frequency domain) and the closed-loop stability properties of APSE.

In selecting the CFD methodologies to model the nozzle dynamics, the importance was placed on selecting methods that model compressible flow, with integration schemes that also seem to preserve dynamic accuracy as opposed to just being time accurate.

\subsubsection{Inlet and Nozzle Control Schedules}

Both the inlet and the nozzle incorporate control schedules to optimize the VCE performance. For the inlet, the center body geometry (for the collapsing centerbody axisymmetric inlet) is adjusted to position the normal shock in the vicinity of the cowllip in order to minimize flow spill and associated drag, while maximizing pressure recovery at supersonic flow conditions. For this normal shock positioning (minimum flow spill), the engine nozzle geometries and flows are also adjusted (via a nozzle control schedule) in order for the engine overall mass flowrate demand to match that captured by the inlet. To satisfy this matching flow demand for the engine, the choked nozzle A8 areas for the main bypass and the core flow are set via a control schedule to satisfy the thrust demand for the particular operating condition, and the VGV schedule of the noise abatement flow path (unchoked nozzle) is the final adjustment for this matching flow demand. At the same time that the A8s are adjusted, the whole nozzle geometry is appropriately changing to properly expand the flow so as to maximize the propulsive efficiency of the nozzles. 
In the past, a nozzle control schedule derivation methodology was developed for a single nozzle to fully expand the flow by utilizing the dynamic engine simulation. This methodology will be covered in a NASA TM version of this paper, which is in progress. For this process, however, NPSS was utilized to derive the control schedules of the VCE nozzles together with the VGV schedule of the noise abatement flow path. The reason is because the process of deriving these control schedules is less laborious by utilizing the NPSS VCE model instead of the corresponding dynamic model. The dynamic VCE model would be used in this case to verify these schedules, but also if necessary to design the stability of the passive feedback loops developed by tying the area control schedules to nozzle PR's. The stability of such passive control loops was dealt with in the methodology developed before for nozzle control schedule derivation, which may also turn out to be a problem with the VCE.

\section{Atmospheric Turbulence Modeling}

A flight vehicle, like a supersonic aircraft, experiences disturbances coming from atmospheric turbulence, and as such, both the propulsion system and the ASE system are susceptible to these kinds of disturbances. In the past, atmospheric type turbulence models have been developed to study the effects of atmospheric disturbances on flying vehicles. The most popular of these models is the so-called Dryden model, ${ }^{16}$ a second order approximation of the actual fractional order of atmospheric turbulence. Under this task, a more accurate model was developed to characterize the natural fractional order of atmospheric turbulence. This model is being utilized to provide atmospheric disturbances for both the propulsion and APSE models.

The atmospheric turbulence model that applies disturbances to the propulsion and the ASE systems is developed by first implementing sinusoidal functions of unit amplitude at discrete frequencies over the frequency range that covers those disturbances, and then summing together the outputs of these sinusoid functions. The frequency range of atmospheric disturbances that influences the vehicle dynamics depends on the speed of the vehicle. ${ }^{17}$ Initially, these spectral sinusoids were implemented with $0^{\circ}$ phase shift and later the phase shift of these sinusoids was randomized. The summation of these spectral sinusoids was utilized as an input to the individual transfer functions (TFs) that constitute the various types of disturbances, such as acoustic velocity, temperature, temperature to acoustic velocity, and pressure disturbances as detailed in the section "Simplified Atmospheric Turbulence Model .." in Ref. 17. These TFs have the eddy dissipation rate as a free parameter, which can be chosen, for instance, based on four times the average value of this parameter recorded in North Atlantic cruise altitudes, or somewhere between this value and the worst case ever recorded. ${ }^{17}$ The output of these TFs through an interface that has been developed provide the appropriate disturbances such as total temperature and pressure, or Mach number, static temperature and pressure, depending on the particular boundary conditions of the propulsion system simulation. For ASE, wind gust disturbances are provided. The density of these spectral sinusoids is also chosen such that the resultant total wind velocity does not exceed a reasonable maximum value for expected wind velocities. ${ }^{17}$

It is assumed for this simulation that atmospheric turbulence generates acoustic velocity wind gusts and static temperature and pressure disturbances based on the atmospheric turbulence model developed in Ref. 17. For the turbulence to propulsion interface with the total temperature and pressure boundary conditions, it is assumed that static temperature disturbances generate additional wind gusts and that these wind gusts also generate additional total pressure disturbances.

\section{APSE Interface models and APSE Integration}

The primary objective of the propulsion system dynamic modeling effort is to develop a propulsion system model that covers the expected frequency range of ASE modes and utilize this model, along with the ASE dynamic model, to develop an integrated vehicle APSE model to study its performance. Thus, a significant subtask of the overall HSP ASE task is the development of APSE models. ${ }^{1}$ An APSE model includes a traditional ASE airframe and aerodynamic mathematical models ${ }^{1}$, a dynamic propulsion system model, and all the relevant interfaces between these models. The development of an APSE model has so far proven to be a rather involved effort, mainly because such models do not currently exist due to the assumption that these two systems do not interact in a significant way, which may not be the case for supersonic vehicles.

Recently, the propulsion and ASE models were integrated to develop a preliminary closed-loop APSE model with the initial purpose to help validate the interfaces and the integration of these models, while checking for the reasonableness of the various responses and providing early performance assessments. In order to integrate these two different types of systems into a closedloop dynamic system, the appropriate interfaces from ASE to propulsion and from propulsion to ASE first needed to be developed. Thus, the modeling approaches for these two interfaces will be discussed before the APSE system is covered.

\subsection{ASE to Propulsion Interface}

An interface model was developed to tie the ASE acceleration measurements to flow field disturbances entering the propulsion system. This was accomplished by integrating the value of an accelerometer measurement located near the leading edge of the wing in the vicinity of the propulsion system (for the under hung engines) to provide for wing velocity, which were then directly associated with flow velocity disturbances. This ASE generated disturbance is then converted to total temperature and pressure deltas that are added to the respective deltas produced by atmospheric turbulence. These combined disturbances are 
then added to the nominal environmental inputs to the engine of total temperature and pressure coming into the inlet as boundary conditions. When the quasi 1D inlet model is integrated with the propulsion system, this interface will change to convert local wing velocity to Mach number, static temperature, and static pressure - the current environmental inputs (boundary conditions) to the quasi $1 \mathrm{D}$ inlet model.

Currently, the ASE system wing displacements are converted to the total temperature and pressure disturbances by employing the total to static relations for ideal gas, compressible, and adiabatic flow to calculate the nominal freestream static temperature and pressure as follows

$$
\begin{gathered}
T_{s}=T_{t}\left(1+\frac{\gamma-1}{2} M^{2}\right)^{-1} \\
P_{s}=P_{t}\left(1+\frac{\gamma-1}{2} M^{2}\right)^{\frac{-\gamma}{\gamma-1}}
\end{gathered}
$$

Utilizing the freestream static temperature, $T_{s}$, the local speed of sound, $a$, and the corresponding delta Mach number, $\Delta M$, produced by the wing velocity, $V_{d}$, can be calculated as follows

$$
\begin{gathered}
a=\sqrt{\gamma R T_{S}} \\
\Delta M=\frac{V_{d}}{a}
\end{gathered}
$$

Utilizing Eq. (12) and Eq. (13), but instead expressing them in terms of the respective total quantities, we can compute their deltas as

$$
\begin{gathered}
\Delta T_{t}=\frac{\partial T_{t}}{\partial M} \frac{\partial M}{\partial t}=T_{S}(\gamma-1) M \Delta M \\
\Delta P_{t}=\frac{\partial P_{t}}{\partial M} \frac{\partial M}{\partial t}=\gamma M P_{S}\left(1+\frac{\gamma-1}{2} M^{2}\right)^{\frac{1}{\gamma-1}} \Delta M
\end{gathered}
$$

Equation (16) and Eq. (17) represent the ASE flow disturbances that are added to the respective atmospheric disturbances to compute the combined freestream disturbances that enter the propulsion system in the APSE simulation.

The same relations are utilized for the atmospheric turbulence to propulsion system interface model to convert atmospheric turbulence of acoustic velocity disturbances to total pressure and temperature deltas. However, in this case, atmospheric turbulence also produces pure temperature and pressure disturbances that, respectively, are added together with the corresponding deltas produce by the acoustic gusts.

The wing accelerations at the vicinity of the propulsion system inlet are mostly in the transverse or vertical direction, while the propulsion system 1D axial flow is in the longitudinal direction. However, it is assumed that the wing leading edge serves to convert tangential disturbances to longitudinal by utilizing a conversion factor. ${ }^{17}$ This approach is applicable to propulsion systems that are mounted under the wing of the vehicle. Similarly, this conversion factor from transverse to longitudinal wind gusts is utilized in the atmospheric turbulence model; as it is assumed that atmospheric acoustic turbulence primarily originates from gravitational waves, ${ }^{17}$ which are transverse type waves. In the future, the plan is to expand the propulsion system model to address flow distortion (2D effect), in which case as appropriate, both transverse and longitudinal disturbances will be utilized.

\subsection{Propulsion to ASE Interface}

The propulsion system produces thrust forces that act on the pylons that connect the engine to the structure of the vehicle. These thrust forces vary with time and can potentially couple with and exacerbate the structural modes of the vehicle. Traditionally, the ASE structural model doesn't include the inputs associated with the thrust forces and the corresponding dynamic couplings. That is because these models have not been utilized before to develop an integrated APSE model.

Recently, the structure of the ASE state space model has been changed to accommodate the thrust forces and their couplings with ASE modes. This was done by modifying the ASE state space model to augment the B-matrix which allows for thrust forces to be used as inputs to the ASE model. Shortly after this change was implemented, tests were conducted at NASA Langley utilizing a semi-span wing model with forces applied at the engine mountings to simulate thrust forces, while measuring the frequency response via accelerometers. Currently, work is ongoing to update the ASE state space model for the thrust interface based on the frequency responses generated from these test measurements. 


\subsection{Integrated APSE Model}

A simplified, proof-of-concept APSE model was developed last year to assess the reasonableness of the interfaces and the closed-loop dynamic behavior of the system, and to also conduct some preliminary performance assessments. A simplified schematic diagram of the major components of this integrated APSE system model for a single engine mounted under an aircraft wing is shown in Fig. 13. The interfaces as discussed before and as shown in Fig. 13 are thrust to ASE and ASE to inlet, as well as atmospheric turbulence to propulsion and ASE. This closed-loop model is expected to behave as a closed-loop dynamic system with typical time and frequency domain responses and stability attributes.

The gust model shown in Fig. 13 separately applies

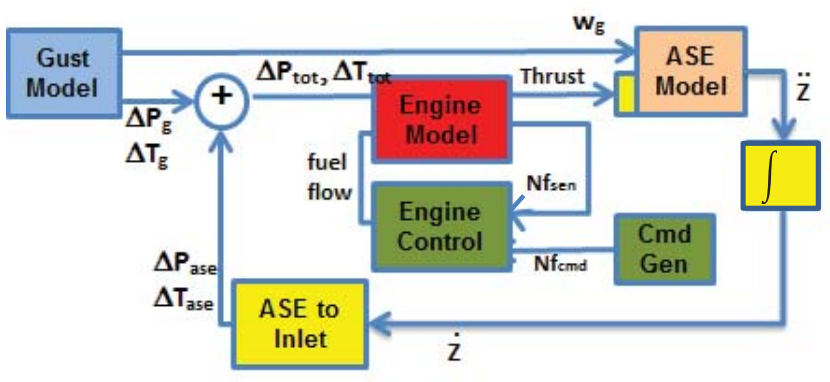

Figure 13. A simplified closed-loop system diagram for an APSE dynamic simulation developed recently.

disturbances to both the propulsion and ASE systems. For the ASE system, these disturbances are in the form of acoustic velocity wind gusts as shown in Fig. 14, while for the propulsion system these disturbances are in the form of total temperature and pressure variations. For this model, given the vehicle speed ( $\mathrm{M} \approx 1.6$ in this case), the disturbance frequencies extend up to 20 Hz. ${ }^{17}$

Simulation results of wing velocity are shown in Fig. 15 (simulation results specifically covering the latest VCE update can be found in Ref. 15). This figure shows the APSE closed-loop wing velocity in the vicinity of the inlet compared to wing velocity with the ASE alone (i.e., without thrust variation forces impacting the wing). The asymmetry in the response in Fig. 15 is due to the asymmetric time domain evolution of atmospheric turbulence, both for the acoustic wind gusts shown in Fig. 14 and for atmospheric pressure-temperature disturbances. These preliminary results indicate that the APSE system may pose a challenge for supersonic vehicle design and that the structural dynamics of the vehicle may be underestimated without including the propulsion dynamics.

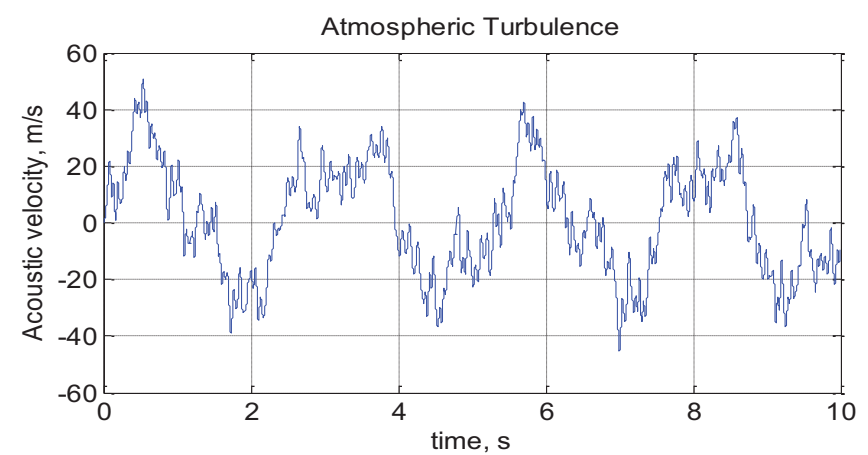

Figure 14. Atmospheric acoustic wing gusts disturbance.

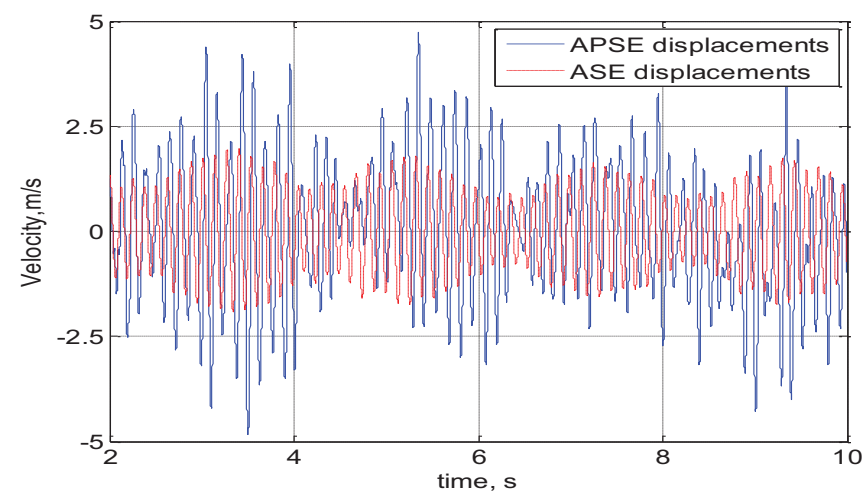

Figure 15. Wing velocity with the propulsion system exerting thrust forces (APSE) and without (ASE alone).

\section{Conclusion}

The development of a dynamic supersonic propulsion system model and subsequent development of an APSE system model to study the dynamic performance of supersonic vehicles is a challenging undertaking. This paper summarizes the work that has been completed to-date for the propulsion system under this effort and the technology areas involved in developing such a model. The approach that has been followed in this effort is to start from simple models and as more is learned, to evolve the complexity of these models to a sufficient state so that in the near future, the challenges associated with APSE can be addressed and be able to answer dynamic performance questions associated with $\mathrm{N}+2$ supersonic vehicles. As it can be seen in the various sections that cover the propulsion system component models, there is no specific model that can be used to answer all the performance questions. Therefore, the development of alternative component models is also covered. These models are tied to the objective and the performance questions that need to be studied. The overall objective is to study closed-loop APSE dynamics, at the frequency range expected for ASE modes, and in this regard, the closed-loop dynamic accuracy of the 
propulsion system models is of utmost importance. Therefore, regardless of the specific purpose of the various component models that are being developed, all these models need to have the necessary dynamic accuracy in the frequency range of interest for both the magnitude and the phase. As currently planned, future efforts will focus on analyzing the overall vehicle dynamics for vehicle stability, ride quality, and aerodynamic efficiency in $1 \mathrm{D}$ flow for the propulsion system as well as 2D/3D to include vehicle maneuvering (pitch, yaw, and roll).

\section{Acknowledgments}

The authors would like to acknowledge the support of the High Speed Project of the NASA Fundamental Aeronautics Program. The authors would also like to acknowledge Kyle Woolwine, a student at The University of Colorado for developing the inlet 2D simulations.

\section{References}

1. Silva, W. A., et al. "The NASA High Speed ASE Project: Computational Analyses of a Low-Boom Supersonic Configuration," AIAA SciTech Conference, Harbor Maryland, Jan. 13-17, 2014.

2. Seldner, K.; Mihaloew, J. R.; Blaha, R. J.; "Generalized Simulation Technique for Turbojet Engine System Analysis," NASA TN D-6610, February 1972.

3. Garrard, D., "ATEC The Aerodynamic Turbine Engine Code for the Analysis of Transient and Dynamic Gas Turbine Engine System Operations Part 1: Model Development," ASME International Gas Turbine Engine Institute Turbo Expo, No. 96-GT-193, 1996.

4. Bisplinghoff, R. L.; Holt, A.; Halfman, R. 1.; “Aeroelasticity,” Dover Publications, 1996.

5. Kopasakis, G.; Connolly, J. W.; Paxson D. E.; Ma P.; "Volume Dynamics Propulsion System Modeling for Supersonic Vehicle Research," GT2008-50524, ASME TurboExpo 2008, Berlin Germany; NASA/TM 215172; Journal of Turbomachinery, Vol. 132, October 2010.

6. Connolly, J.; Kopasakis, G.; Paxson, D.; Stuber, E.; Woolwine, K.; "Nonlinear Dynamic Modeling and Controls Development for Supersonic Propulsion System Research," $48^{\text {th }}$ AIAA/ASME/SAE/ASEE Joint Propulsion Conference and Exhibit, AIAA 2011-5635, August, 2011.

7. Mattingly, J. D.; Heiser, W. H.; Pratt, D. T.; "Aircraft Engine Design Second Edition," AIAA Education Series, Published by American Institute of Aeronautics and Astronautics, Inc., Reston, VA, 2002.

8. Willob, R. G.; Seldner, K.; "Multistage Compressor Simulation Applied to the Prediction of Axial Flow Instabilities," NASA TM X-1880, Sept. 1969.

9. Greitzer, E. M.; "Surge and Rotating Stall in Axial Flow Compressors," Transactions of the ASME, pp. 190-198. Surge physics, April, 1976.

10. O'Brien, W. F., Dynamic Simulation of Compressor and Gas Turbine Performance, May, 1992, AGARD Lecture Series LS183.

11. Varner, M.O., Martindale, W.J. Phares, K.R, Kneile, K.R., Adams, J.C., "Large Perturbation Flow Field Analysis and Simulation for Supersonic Inlets Final Report," NASA CR 174676.

12. Kopasakis, G.: Connolly, J. W.: Paxson, D. E.: Woolwine, K: "Quasi 1D Modeling of Mixed Compression Supersonic Inlets," 50 ${ }^{\text {th }}$ Aerospace Science Meeting, Nashville, TN, AIAA-2012-0775, January 2012.

13. Kopasakis, G.; Connolly, J. W.; Kratz, J.; "Quasi One-Dimensional Unsteady Modeling of External Compression Supersonic Inlets," $48^{\text {th }}$ AIAA/ASME/SAE/ASEE Joint propulsion Conference \& Exhibit, AIAA 2012-4147, Atlanta, GA, 30 July - Aug. 1, 2012.

14. Anderson, J. D. Jr.; "Computational Fluid Dynamics - The Basics with Applications," McGraw-Hill, Inc., 1995.

15. Connolly, J. W.; Kopasakis, G.; Friedlander, D.; "Computational Fluid Dynamics Modeling of a Supersonic Nozzle and Integration into a Variable Cycle Engine Model,” AIAA Joint Propulsion Conference, Cleveland, Ohio, July 28-30, 2014 (publication pending).

16. Hoblit, F. M.: "Gust Loads on Aircraft: Concepts and Applications," AIAA Education Series, 1988.

17. Kopasakis, G.; "Modeling of Atmospheric Turbulence as Disturbances for Control Design and Evaluation of High Speed Propulsion Systems," ASME Journal of Dynamic Systems, Measurement, And Control, Vol. 134, March 2012. 